\title{
Analytical Approximations to the Dynamics of Superparabolic Level Crossing Models
}

\author{
Chon-Fai Kam ${ }^{1}$ and Yang Chen ${ }^{1}$ \\ ${ }^{1}$ University of Macau
}

August 17, 2020

\begin{abstract}
We study the dynamics of a two-level crossing model with a cubic modification of the linear Landau-Zener tunneling, and express the non-adiabatic transition amplitudes in terms of the bi-confluent Heun functions which are generalizations of the confluent hypergeometric functions. We find a closed-form series expression of the transition probability at the long time limit, and derive tractable approximate formulas to the state populations by use of simple functions in a large part of the parameter space. The analytical approximations are validated by comparison with numerical results.
\end{abstract}

\section{Hosted file}

Analytical Approximations to the Dynamics of Superparabolic Level Crossing Models.pdf available at https://authorea.com/users/351537/articles/476114-analytical-approximations-tothe-dynamics-of-superparabolic-level-crossing-models 